\title{
Effective temperature of the sun based on log periodic dipole antenna performance in the range from $45 \mathrm{mhz}$ to $870 \mathrm{mhz}$
}

\author{
Z. S. Hamidi 1,3,", S. H. Chumiran ${ }^{2}$, A. Mohamad ${ }^{4}$, N. N. M. Shariff ${ }^{3}$, Z. A. Ibrahim ${ }^{3}$, \\ N. N. Radzin ${ }^{1}$, N. Hamzan ${ }^{1}$, N. M. Anim ${ }^{3}$, A. N. Alias ${ }^{2}$ \\ ${ }^{1}$ Faculty of Applied Sciences, MARA University of Technology, UiTM S.Alam Selangor \\ ${ }^{2}$ Faculty of Applied Sciences, MARA University of Technology, UiTM P.Pinang \\ ${ }^{3}$ Faculty of Science, University Malaya, Kuala Lumpur, Malaysia \\ ${ }^{4}$ Faculty of Electrical Engineering, MARA University of Technology, UiTM P.Pinang \\ Email address: \\ zetysh@salam.uitm.edu.my (Z. S. Hamidi)
}

\section{To cite this article:}

Z. S. Hamidi, S. H. Chumiran, A. Mohamad, N. N. M. Shariff, Z. A. Ibrahim, Z. Z. Abidin, N. N. Radzin, N. Hamzan, N. M.Anim, A.N. Alias. Effective Temperature of the Sun Based on Log Periodic Dipole Antenna Performance In The Range From $45 \mathrm{MHz}$ to $870 \mathrm{MHz}$, American Journal of Modern Physics. Vol. 2, No. 2, 2013, pp. 58-60. doi: 10.11648/j.ajmp.20130202.14

\begin{abstract}
The Log Periodic Dipole Antenna (LPDA) performance of solar burst monitoring in the range of (45 -870) MHz is presented. This project is under the International Space Weather Initiative (ISWI) program in order to observe the solar activities within 12 hours per day. At the first stage, we make an effort by constructing LPDA that suitable with the criteria, specification and practical enough with 5.45 meter boom length size of the antenna. The rods then were perforated with nineteen (19) elements that were made of aluminum rods. Two (2) rod aluminums type with nineteenth (19) elements that signify different frequencies. The gain of this antenna is 6.67 which is required preamplifier to amplify the signal of the Sun. From the results, we found that the effective temperature of the antenna is $1.3 \mathrm{~dB}$ and the temperature of the Sun during that observation is $1578 \mathrm{~K}$. Some suggestions on improving this design are also presented.
\end{abstract}

Keywords: Log Periodic Dipole Antenna (LPDA), Sun, Solar Burst, Effective Temperature

\section{Introduction}

Log Periodic Dipole Antenna (LPDA) is one type of antenna that requires an $\mathrm{N}$ number of elements with different size to drive the voltage loaded by arbitrary reactors at each center. It was invented by Dwight E. Isabell with each element represents a different frequency with alternating element with $180^{\circ}$ [1]. One the advantage of LPDA is the boom length of the antenna enhances a greater performance especially in gain compare Yagi antenna [2]. However, it might be too long depends on the range of the frequency.

Previous work had been done by [3] discovered the potential sites to build up a radio observatory in Malaysia whereby National Space Centre is one of the candidate sites. Due to geophysical factor, Malaysia have a good potential in monitoring solar activities in radio region with 12 hours monitoring daily throughout a year. Situated close to the equator and received almost 12 hours of sunlight has given Malaysia a good merit to monitor solar activities daily throughout a year in radio region. This has opened up opportunities for international collaboration. CALLISTO (Compound Astronomical Low Frequency Low Cost Instrument for Spectroscopy Transportable Observatory) network is one of the International Space of Weather Initiative Program (ISWI) project to study the magnetic field of the Sun. Based on this prospect, we make an initiative to construct the Log Periodic Dipole Antenna (LPDA) that suitable with the criteria, specification and practical enough with 5.45 meter boom length size of the antenna. In this work, we evaluate the performance of LPDA that is being constructed in January 2012. This antenna has successfully set up at National Space Centre, Banting on 15th February 2012. We make an initiative by determining the effective area of the antenna and the performance of the antenna can be evaluated by connecting to the CALLISTO spectrometer.

\section{Effective Area of Antenna}


Turning to solar observation, the surprising development of observational tools has greatly contributed to our understanding of magnetic and gaseous fine structure of solar bursts [4]. Radiation from the quiet Sun is produced by the thermal mechanism of bremsstrahlung, and radio bursts of several kinds are produced by the non-thermal mechanisms of plasma radiation and, rarely, gyro synchrotron radiation can be detected by using LPDA [5]. Certain phenomena such as Coronal mass ejections (CMEs) are composed of multi-thermal plasmas, which make them produce different radio signatures at different wavelengths [6]. It is important to have a proper method in order to ensure a standard and accuracy measurement of analysis the solar data sets and make comparable at different sites [7]. The procedure for measuring the flux density of radio flux emanating from a discrete source consists in directing the log periodic antenna to the Sun. Once the receiving is calibrated, the deflection may be converted into Log Periodic Dipole Antenna (LPDA) temperature, knowing as gain and it is possible to obtain as spectral flux density in Watt/ meter ${ }^{2}$ cycle second. Here, we discussed some important parameter and aspect that need to be considered. Since the electronic calibration is not available, therefore the calibration was done indirectly using quiet Sun flux. From earlier experiments we recognize the efficiency factor $\eta=0.34$ of the antenna system. Thus the effective aperture is:

$$
A_{e f f}=A_{g e o} \eta=\pi \eta^{2}=6.7 \mathrm{~m}^{2}
$$

Meanwhile, the Y-factor of the Sun can be determined by:

$$
Y_{\text {sun }}=\frac{I_{\text {sun }}}{I_{\text {sky }}}=\frac{67396}{13671}=4.93 \mathrm{~dB}
$$

Similarly, the Y-factor of the radio frequency interference (RFI) also signal can be determined by:

$$
Y_{r f i}=\frac{I_{r f i}}{I_{\text {sun }}}=\frac{74215}{67396}=1.10 \mathrm{~dB}
$$

The results of this study indicate that the antenna temperature Tant of the Sun to:

$$
T_{\text {Sun }}=\frac{S_{\text {Sun }} A_{e f f}}{2 k}=1578 \mathrm{~K}
$$

It will lead us to calculate the system temperature Tsys:

$$
T_{\text {sys }}=\frac{T_{\text {Sun }}}{Y_{\text {Sun }}-1}=402 \mathrm{~K} \rightarrow N F=1.3 \mathrm{~dB}
$$

This result indicates that the correlation between temperature of the antenna and the Sun.

\section{Specification of LPDA and Observa- tions}

It is well known that the design of a log- periodic dielectric resonator antenna consists of a basic geometric pattern that repeats, except with a different size pattern [8]. In order to obtain more gain than with a single log-periodic array, two (2) aluminum rods were chosen due to its properties as non-corrosive and light weight [9]. The feed point for the array is seen to increase smoothly in dimension, being greater for each element than the previous element in the array. However, for frequency-independent operation, Rumsey's principle requires that the locations of all elements be specified by angles rather than distances. Therefore, for very wide bandwidths, the log periodic dipole array must be correspondingly longer. At the receiving antenna, the electromagnetic energy is collected and converted into electrical energy and fed to the receiver. RG58 coaxial cables are used for feeding the outdoor antennas and we use either the radio frequency (RF) transformers for impedance transformation between the antenna and the cable. Here, we designed and constructed the log periodic dipole antenna with the range of frequency from $45 \mathrm{MHz}$ till $870 \mathrm{MHz}$ [10]. The main observation system consists of a log periodic dipole antenna, CALLISTO spectrometer and computer connected to the internet. For standardized the time, GPS clock is used to control the sampling time of the spectrometer and a tracking controller control the antenna direction. The observations will start from 07:30am till 19:30 pm, 12 hours every day. Table 1 shows the physical specification of LPDA.

Table 1. Physical specification of LPDA.

\begin{tabular}{ll}
\hline Frequency range & $45 \mathrm{MHz}$ to $870 \mathrm{MHz}$ \\
Polarization & Linear \\
Input impedance & $50 \Omega$ (unbalanced) \\
Standing wave ratio (VSWR) & $1 \leq 2$ \\
Gain & $6.67 \mathrm{dBi}$ \\
Front-to-back ratio & $>25 \mathrm{~dB}$ \\
RF connector & $\mathrm{N} \mathrm{connector}$ \\
Dimensions & $18 \mathrm{~mm} \mathrm{x} 18 \mathrm{~mm}$ \\
Antenna length & $5.45 \mathrm{~m}$ \\
Max. antenna width & $3.96 \mathrm{~m}$ \\
Weight & $6.5 \mathrm{~kg}$ \\
Humidity & $+55^{\circ} \mathrm{C} / 95 \%$ \\
\hline
\end{tabular}

Table 2. Specification elements of Log Periodic Dipole Antenna.

\begin{tabular}{llll}
\hline $\begin{array}{l}\text { No of } \\
\text { Elements }\end{array}$ & $\mathbf{L}(\mathbf{m})$ & $\mathbf{R}(\mathbf{m})$ & $\mathbf{D}(\mathbf{m})$ \\
1 & 3.96533329 & 5.551466963 & 1.110293326 \\
2 & 3.17226668 & 4.441173636 & 0.888234674 \\
3 & 2.53781338 & 3.552938962 & 0.710587750 \\
4 & 2.03025073 & 2.842351212 & 0.568470208 \\
\hline
\end{tabular}




\begin{tabular}{llll}
\hline 5 & 1.62420061 & 2.273881003 & 0.454776173 \\
6 & 1.29936050 & 1.819104830 & 0.363820944 \\
7 & 1.03948842 & 1.455283885 & 0.291056759 \\
8 & 0.83159075 & 1.164227125 & $0 . .232845411$ \\
9 & 0.66527261 & 1.164227125 & 0.186276331 \\
10 & 0.53221809 & 0.745105382 & 0.149021067 \\
11 & 0.42577448 & 0.596084315 & 0.119216855 \\
12 & 0.34061959 & 0.476867459 & 0.095373486 \\
13 & 0.27249567 & 0.381493973 & 0.076298790 \\
14 & 0.21799654 & 0.305195182 & 0.061039032 \\
15 & 0.17439723 & 0.244156150 & 0.048831227 \\
16 & 0.13951779 & 0.195324922 & 0.039064982 \\
17 & 0.11161423 & 0.156259940 & 0.031251986 \\
18 & 0.08929139 & 0.125007954 & 0.025001589 \\
19 & 0.07143311 & 0.100006365 & 0.020001271 \\
\hline
\end{tabular}

\section{Discussion and Conclusion}

The band from $45 \mathrm{MHz}$ to $870 \mathrm{MHz}$ has the longest boom length of $5.45 \mathrm{~m}$. As a consequence, the longest dipole in the array will be $3.96 \mathrm{~m}$ long. In case of array, elements of proper geometry can be assembled and fed in a suitable way [11] In addition, the diameter of $18 \mathrm{~mm}$ for the dipoles makes $Z_{a}$ becomes $14.43 \mathrm{~W}$. The estimated gain of our LPDA is $6.67 \mathrm{~dB}$ for relative spacing constant $(\sigma)$ and the ratio of the length $(\tau)$ of 0.14 and 0.8 , respectively. By LPDA input impedance $\left(\mathrm{R}_{0}\right)$ of $50 \mathrm{~W}$, the parallel rod feeder impedance $\left(\mathrm{Z}_{0}\right)$ is approximately $223 \mathrm{~W}$. We also found that higher input impedance would have an adverse effect on the spacing of the booms.

In this work, the construction and performance of the temperature antenna of Log Periodic Dipole Antenna (LPDA) have been discussed. It has been successfully set up at the National Space Agency, Banting Selangor in February 2012. However, in order to reduce noise, we also strongly recommend the installation of this antenna as far as possible from Radio Frequency Interference (RFI) sources. Yet, there are still needs to be improved especially in optimization the gain of the signal and modification of electrical, mechanical and mounting aspect. We hope that we could improvise the construction of the LPDA in the next project to be installed at three (3) different sites which are MARA University of
Technology, University of Malaya and in Jelebu observatory.

\section{Acknowledgements}

We thank the anonymous referee for helpful comments which greatly improved and clarified this work. Solar monitoring is a project of cooperation between UiTM and University Malaya. The author also would like to thank to C. Monstein from Switzerland and Prof Dr Zainol Abidin Ibrahim for the technical part of advice. This research has made use of Radio Astrophysics and Cosmology Lab Facility. This work was partially supported by the FRGS $(600$ RMI/FRGS 5/3 2012) UiTM and PPP UM PV071/2011B grants.

\section{References}

[1] G.H. Zhai, W. Hong, K. Wu, Z.Q. Kuai, Wideband substrate integrated printed log-periodic dipole array antenna, IET Microw. Antennas Propag. 4 (2010) 899-905.

[2] R. Carrel, The design of log-periodic dipole antennas, IRE International Convention Record, 1961, pp. 61- 75.

[3] Z.Z.e.a. Abidin, Investigation of Radio astronomical windows between $1 \mathrm{MHz}-2060 \mathrm{MHz}$ in University of Malaya, New Astron. 14 (2009) 579-583.

[4] Arnold O. Benz, C. Monstein, a.H. Meyer, CALLISTO, A New Concept for Solar Radio Spectrometers, Kluwer Academic Publishers, 2004.

[5] D.J. McLean, a.N.R. Labrum, Solar Radiophysics, Cambridge University Press, Cambridge, 1985.

[6] N. Gopalswamy Geophysical Monograph Series 165 (2006).

[7] Z. Hamidi, Z. Abidin, Z. Ibrahim, N. Shariff, U.F.S.U. Ibrahim, R. Umar, Preliminary analysis of investigation Radio Frequency Interference (RFI) profile analysis at Universiti Teknologi MARA, IEEE, 2011, pp. 311-313.

[8] J. Carr, Some variations in log-periodic antenna structures, IRE Trans. Antennas Propag., 1961, pp. 229- 230.

[9] Z. Hamidi, Z. Ibrahim, Z. Abidin, M. Maulud, N. Radzin, N. Hamzan, N. Anim, N. Shariff, Designing and Constructing Log Periodic Dipole Antenna to Monitor Solar Radio Burst: e-Callisto Space Weather, (2012).

[10] Z. Hamidi, Z. Abidin, Z. Ibrahim, C. Monstein, N. Shariff, Signal Detection Performed by Log Periodic Dipole Antenna (LPDA) in Solar Monitoring, (2012).

[11] M.S.M. Aras, M.K.A. Rahim, Z.Rasin, M.Z.A.A. Aziz, An Array of Dielectric Resonator Antenna for wireless application, IEEE International RF and Microwave, 2008, pp. 459.463 . 